\title{
Discrete Set Person Name Data Type
}

National Cancer Institute

\section{Source}

National Cancer Institute. Discrete Set Person Name Data Type. NCI Thesaurus. Code C95691.

A data type comprised of a collection of a discrete set of person names. 